\title{
Exploring the Modeling of Declarative Processes Using a Hybrid Approach
}

\author{
Abbad Andaloussi, Amine; Buch-Lorentsen, Jon; Lopez, Hugo A.; Slaats, Tijs; Weber, Barbara
}

Published in:

Conceptual Modeling.

Link to article, DOI:

10.1007/978-3-030-33223-5_14

Publication date:

2019

Document Version

Peer reviewed version

Link back to DTU Orbit

Citation (APA):

Abbad Andaloussi, A., Buch-Lorentsen, J., Lopez, H. A., Slaats, T., \& Weber, B. (2019). Exploring the Modeling of Declarative Processes Using a Hybrid Approach. In Conceptual Modeling. (pp. 162-70). Springer. Lecture Notes in Computer Science Vol. 11788 https://doi.org/10.1007/978-3-030-33223-5 14

\section{General rights}

Copyright and moral rights for the publications made accessible in the public portal are retained by the authors and/or other copyright owners and it is a condition of accessing publications that users recognise and abide by the legal requirements associated with these rights.

- Users may download and print one copy of any publication from the public portal for the purpose of private study or research.

- You may not further distribute the material or use it for any profit-making activity or commercial gain

- You may freely distribute the URL identifying the publication in the public portal 


\title{
Exploring the Modeling of Declarative Processes Using a Hybrid Approach
}

\author{
Amine Abbad Andaloussi ${ }^{1}$, Jon Buch-Lorentsen ${ }^{1}$, Hugo A. López ${ }^{2,5}$, Tijs \\ Slaats $^{3}$, and Barbara Weber ${ }^{1,4}$ * \\ 1 Software and Process Engineering, Technical University of Denmark \\ 2800 Kgs. Lyngby, Denmark - amab@dtu.dk \\ 2 Department of Computer Science, IT University of Copenhagen, Denmark \\ 3 Department of Computer Science, University of Copenhagen, Denmark \\ ${ }^{4}$ Institute of Computer Science, University of St. Gallen, Switzerland \\ ${ }^{5}$ DCR Solutions A/S, Denmark
}

\begin{abstract}
Process modeling aims at providing an external representation of a business process in the shape of a process model. The complexity of the modeling language, the usability of the modeling tool, and the expertise of the modeler are among the key factors defining the difficulty of a modeling task. Following a qualitative analysis approach, this work explores a hybrid modeling technique enhanced with a tool (i.e., the Highlighter) to guide the transition from informal text-based process descriptions to formal declarative process models. The exploratory results suggest that this technique provides cognitive support to modelers and hint towards an enhanced quality of process models in terms of alignment, traceability of process requirements and availability of documentation. The outcome of this work shows a clear opportunity for future work and provides a framework for further empirical studies.
\end{abstract}

\section{Introduction}

A process model is a visual/graphical representation of the different components of a business process, as well as their interrelations. The full understanding of a process tends to be a joint construction between different process design artifacts (process artifacts for short), including the business process model. In this paper, we examine an approach used to relate textual process artifacts and business process models during the Process of Process Modeling (PPM for short). This process is regarded as a "design activity" where a modeler develops an internal representation of the business process and externalizes it through one or many process artifacts [3]. Throughout this process, three levels of cognitive load are induced. (1) Intrinsic load is associated with the complexity of the material being processed, while (2) extraneous load is rising from the unnecessary

\footnotetext{
* Work supported by the Innovation Fund Denmark project EcoKnow (7050-00034A), the Danish Council for Independent Research project Hybrid Business Process Management Technologies (DFF-6111-00337), and the European Union's Horizon 2020 research and innovation programme under the Marie Sklodowska-Curie grant agreement BehAPI No.778233.
} 
representational complexity of the task. (3) Germane load, in turn, is associated with the effort invested in building an appropriate scheme to organize new information efficiently [5]. During a modeling session, intrinsic load emerges from the complexity of inferring a mental model from a set of process specifications. Extraneous load raises from the formulation of the textual process description and the complexity of the modeling tool. While intrinsic load is inherent to the task and thus unavoidable, efforts can be made to reduce the extraneous load by improving the quality of the tool-support and enhancing the PPM experience.

When considering the declarative modeling paradigm, the requirement for lowering extraneous load in favor of extra intrinsic processing becomes more stringent. This is due to the understandability of declarative languages, which is shown to be controversial especially for novice end-users [8]. A hybrid modeling approach can, in turn, be used to facilitate the modeling of declarative business processes and provide additional channels to support the PPM through a set of interrelated process artifacts. In this vein, the Highlighter [11] was introduced.

The Highlighter (cf. Fig. 1b) is integrated with the default Dynamic Condition Response (DCR [10]) graphical modeling tool (shortly, the Modeler, cf. Fig. 1a) and a guided simulation (cf. Fig. 1c). The tool displays a process model and an annotatable textual description side-by-side allowing to map the specifications in the textual process description with the corresponding model elements (i.e., activities, roles and relations). During a typical modeling session, end-users can design process models by highlighting activities, roles and relations in the process description, then intertwine with the Modeler and the guided simulation to reconcile and validate the process model. Following a qualitative research approach, this work aims at exploring the understandability of such a hybrid process artifact. The remainder of this paper is organized as follows. Sect. 2 provides an overview of the existing hybrid process artifacts. Sect. 3 explains the research method. Sect. 4 reports the obtained findings. Sect. 5 provides a discussion, while Sect. 6 wraps up the key findings and presents future work.

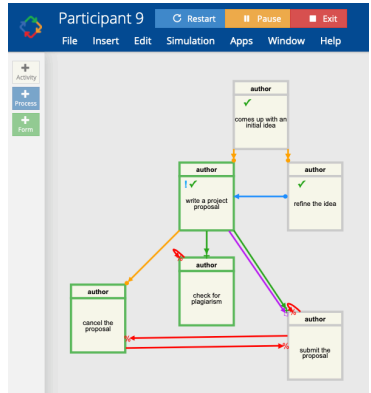

(a) DCR Modeler

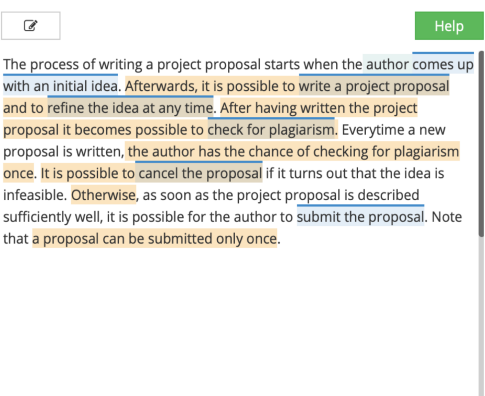

(b) Highlighter

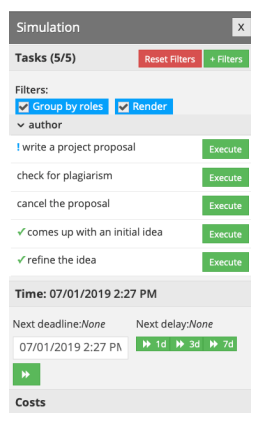

(c) Simulator

Fig. 1: A hybrid process artifact combining the Modeler, the Highlighter and the simulation tools. Available online as part of the DCR platform at https://dcrgraphs.net/ 


\section{Background and Related Work}

Hybrid process representations are introduced in the literature in two contexts: (a) to designate hybrid languages (e.g., [14]) or (b) to describe hybrid process artifacts. While hybrid languages combine existing languages to enable a concise and precise representation of business processes, hybrid process artifacts combine two or more process artifacts overlapping in the description of some aspects of the business process [1]. The emergence of hybrid process artifacts is driven by three main motivations: (1) supporting the understandability of process models (cf., [1,2]), (2) enhancing the maintainability of process models (cf., [16]), and (3) improving the modeling of business processes.

Similar to this work, Dengler and Denny, in [7], propose a hybrid process artifact that combines process models and textual descriptions, embedded in a wiki-based platform. The proposed representation aims at improving the PPM experience by enabling different stakeholders to extract process knowledge and to express business processes using both formal and informal constructs. The findings of a qualitative analysis show that the proposed approach supports better knowledge elicitation. With the same idea in mind, Pinggera et al. in [12] propose the Literate Process Modeling (LiProMo) approach aiming at interweaving annotations and graphical process models to enhance the communication when modeling business processes.

\section{Research Method}

This section introduces the research questions, presents the subjects who took part in this study, describes the material and the procedure followed to run the study and explains the approach used to analyze the collected data.

Research Questions: The Highlighter aims at enhancing the PPM experience by providing a tool-support allowing to facilitate the transition from a textual process description to a graphical process model. In order to investigate this support, it is necessary to understand the way the Highlighter is used in practice. To this end, the first research question is formulated as follows: RQ1: How do users engage with a modeling task using the Process Highlighter?

By enhancing the PPM experience, the Highlighter is expected to positively affect the perceived quality of the produced models. To explore this angle, the second research question is formulated as follows: RQ2: In what aspects can the Highlighter help to improve the quality of process models?

Participants The participants who took part in this study included novice subjects from industrial and education environments. In the former, 7 employees from the Syddjurs municipality in Denmark, and from the latter, 10 students from the Technical University of Denmark (DTU).

Material The material used to conduct this study originates from a process introduced by Reichert and Weber in [13][p. 349]. This process describes the 
writing of a project proposal. The material was presented in Danish at Syddjurs municipality and in English at DTU. A copy of the material is available online at http://andaloussi.org/papers/ER2019/material.pdf

Procedure The study was conducted in both Syddjurs municipality and DTU. Participants were introduced to the modeling notation and the use of the Highlighter in both locations. Then, participants were given a familiarization task on PPM using the tool and the notation. Next, the participants were given the description of the project proposal process and were asked to use the Highlighter to derive the corresponding process model. We collected participant's insights about their experience with the tool from retrospective think-aloud sessions.

Analysis Approach In order to address our research questions, two different analyses have been performed. At the first stage, we have extracted the interactions of the users with the DCR modeling platform. This data were filtered to keep only the interactions associated with adding activities, roles and relations. Next, these interactions were split between those using the Highlighter, and those using the Modeler. During the analysis, the interactions were aggregated over all the modeling sessions and projected according to their time-occurrence into a rhythm eye chart [9]. An example of such a visualization is shown in Fig. 2. The ring structure represents a time-line, the different percentages refer to the progress in relative time. Events (i.e., interactions) are projected as thin lines onto the ring and events of similar type (e.g., interaction with the Highlighter) are depicted with the same color. Besides the user interactions, the collected verbal data were transcribed and analyzed following a qualitative coding approach based on concepts from grounded theory [6].

\section{Findings}

This section reports the findings. Sect. 4.1 scrutinizes the way users engage with a modeling task using the Highlighter. Sect. 4.2 explores whether the proposed hybrid modeling approach can improve the quality of process models.

\subsection{How do Users Engage With a Modeling Task Using the Process Highlighter? (RQ1)}

The users' interactions collected throughout the modeling sessions provide deepened insights into the way end-users engaged with the Highlighter. As shown in Fig. 2, most of the interactions with the Highlighter occurred during the first quarter of the modeling session, which in turn, suggests that most end-users initiated the modeling using the Highlighter and then progressively moved to the Modeler. To further substantiate this modeling pattern, the users' interactions were scrutinized to identify the common interactions within each of the process artifacts. As shown in Fig. 3a, a larger portion of activities were appended to the model using the Highlighter. Similarly, Fig. 3b shows that most roles were added using the Highlighter. Unlike activities and roles, Fig. $3 \mathrm{c}$ shows that relations were mostly added using the Modeler, which in turn suggests that the 


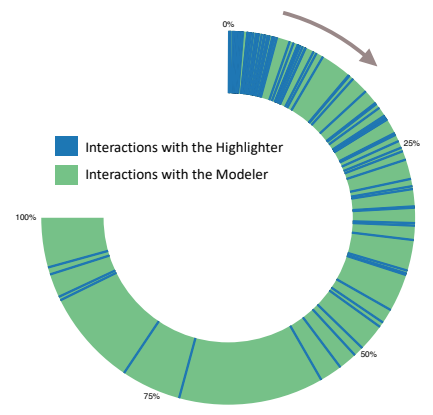

Fig. 2: The interactions associated with the Highlighter and the Modeler tool.

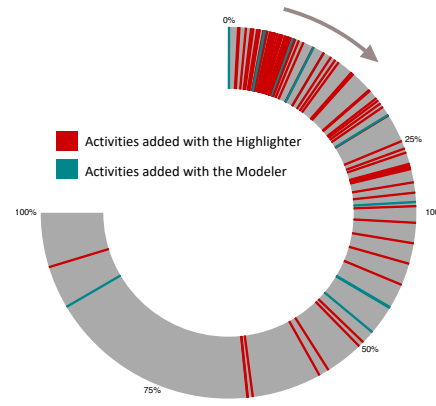

(a) Adding activities

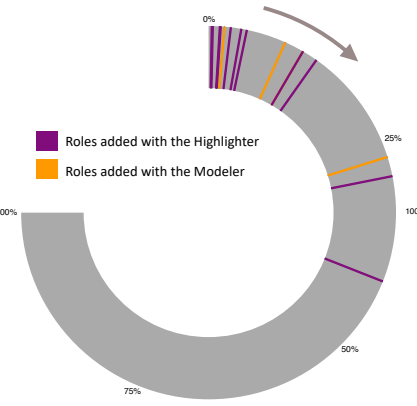

(b) Adding roles

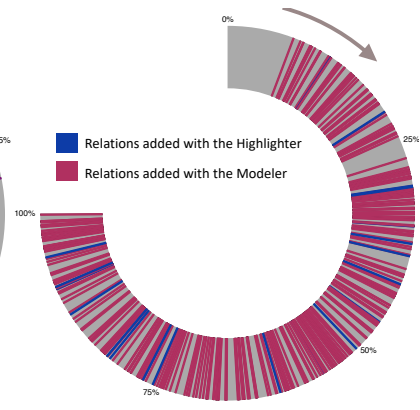

(c) Adding relations

Fig. 3: Interactions associated with adding activities, roles and relations.

Highlighter was not extensively used to add relations. These users' interactions come in line with the subjective insights provided by the participants during the think-aloud. Indeed, most participants affirmed using the Highlighter to identify activities and roles from the process description and resort to the Modeler to add relations. These insights raise the following questions: (1) Why is the Highlighter perceived more efficient to identify and add activities and roles? (2) What makes the use of the Modeler tool more attractive for adding relations to the model?

To answer both questions, we turn to the qualitative coding of the verbal data. In respect to (1), the participants mentioned that the tool provides a kickstart to process modeling and helps in developing an overview of the business process (e.g., "Definitely, I think it is way easier to use the Highlighter to create the activities and it gives a better overview"). Moreover, some participants have associated the use of the Highlighter with its ability to provide structure and to decompose the complexity of the process description (e.g., "it is [referring to the Highlighter] a nice way to structure the text"). Other participants mentioned that the Highlighter can help to memorize the process specifications and to draw attention to specific fragments of the process description (e.g., "It was faster that was the main focus. at least I feel that [it] helps speed things up. I did not really notice that text was highlighted because I already knew what I had highlighted myself, so I mainly focused on the relations that could be between them"). 
In respect to (2), while the identification of activities and roles was straightforward for most participants, many of them faced difficulty when trying to add relations in the Highlighter. Some participants justified their abstention with the argument that the Modeler tool provides a two-dimensional visualization allowing to perceive the interplay between the different activities (e.g., "It just seemed easier once the visual aspect of the activities were done, then you could just connect them directly"). In addition, some participants struggled to locate the exact textual fragment referring explicitly to a specific constraint in the process description. This struggle might be due to the phrasing of the process description (e.g., "For the relations, I'm not sure it's the problem of the Highlighter or on the formulation of the text"). Unlike activities and roles which are often explicit in the process description, relations may not be always explicit in the text.

\subsection{In What Aspects Can the Highlighter Help to Improve the Quality of Process Models? (RQ2)}

From the think-aloud, it has emerged that the mapping between the process model and the process description supports better traceability of the process specification (e.g., "Using the Highlighter makes sense in the sense that it adds traceability ... it helped me map the relations to the requirements") and enables a wider coverage of the requirements in the process description (e.g., "It would be useful after and it is also useful during because I can see whether I already covered some piece of text"). In addition, the participants' quotes indicate that the Highlighter was used to check the alignment between the process description and the process model (e.g., "It [referring to the Highlighter] becomes indispensable as a method to verify whether the process fits with what has been described"6). Last but not least, some participants emphasized the importance of using the Highlighter as a mans to document their process models (e.g., "I think it is very useful as a documentation tool and documentation can also be very useful during the process"). Indeed, the explicit links between the process model and the textual process description can serve for documenting the semantics of the model and enabling modelers to justify their modeling choices [12].

\section{Discussion}

The findings of this exploratory study provide several indications about the perceived benefits of the Highlighter. Both the subjective insights obtained from the participants and the user interactions extracted from the modeling platform show that the Highlighter was perceived more efficient to identify and append activities and roles to the model. These insights fall in line with the conclusions drawn from cognitive psychology. Indeed the use of the Highlighter to markup specific fragments of the process description (e.g., activities, roles) can be associated with a well-known phenomenon referred in cognitive psychology as the isolation effect [15]. This effect is shown to increase the reader attention on

\footnotetext{
${ }^{6}$ Quote translated from Danish
} 
specific parts of the text and help memorizing them [4] [15]. This, in turn, can potentially explain the participants' insights related to the increased memory and attention when using the Highlighter and to some extent support the other insights about the ability of the Highlighter to provide overview and structure as well as to reduce the complexity of the process description (cf. Section 4.1). In addition to that, the quotes of several participants indicate that the Highlighter can support increased traceability, enhanced coverage and better alignment between the process model and the corresponding process description. However, when it gets to identify relations in the model, the Highlighter was challenging. As mentioned in Section 4.1, This challenge is associated with the difficulty in identifying the right text reflecting a certain constraint in the process model, which can be due to the phrasing of the process description.

All these insights provide indications about the extraneous load arising from using the tool. Indeed, the cognitive support provided by the Highlighter can reduce the complexity of the modeling task and contribute to an enhanced PPM experience. However, the implicitness of some constraints in the process description can add an extra layer of complexity when trying to map them to DCR relations, which in turn can induce a higher extraneous load. Hence, the use of the Highlighter can be presumably more effective with process descriptions comprising explicit constraints.

Finally, it has to be noted that the outcome of this exploratory work can be subject to limitations mainly with regards to the number of participants who participated in the study. Therefore, it is hard to generalize the reported findings and draw strong conclusions about the use of hybrid process artifacts in general and the Highlighter in particular. Nevertheless, the outcome of this work provides interesting insights emerging from the users' experience and sheds light on the direction of subsequent empirical investigations.

\section{Conclusion and Future Work}

This work summarizes the findings of an exploratory study investigating the modeling of DCR graphs with the support of the Highlighter. The results suggest that the use of the Highlighter is associated with increased support in PPM and hints toward an enhanced quality of process models. The outcome of this study provides strong indications for the direction of future work. Based on the conclusions drawn from cognitive psychology, we hypothesize that (a) the Highlighter reduces the cognitive load induced during a modeling task. Moreover, following the insights about the explicit mapping between the process specifications and the corresponding model elements we hypothesize that (b) the Highlighter improves model comprehension and clarifies the semantics of the model. Concerning the quality of process models, we hypothesize that (c) the Highlighter provides better alignment between the process description and the process model and enables covering the majority of the requirements mentioned in the text.

These hypotheses define our direction for future work. Following a quantitative analysis approach, we are planning a series of experiments to test and 
validate each of these hypotheses in controlled experimental settings. Moreover, it would be worth to investigate in the up-coming studies the support offered by the Highlighter when integrated with other process modeling languages from both the declarative and the imperative paradigms. The findings will serve as a basis to validate the usability of the Highlighter and will help to improve the design of similar hybrid process artifacts.

\section{References}

1. A. Abbad Andaloussi, A. Burattin, T. Slaats, C. A. Petersen, T. T. Hildebrandt, and B. Weber. Exploring the Understandability of a Hybrid Process Design Artifact Based on DCR Graphs. In BPMDS, LNBIP, pages 1-15. Springer, 2019.

2. A. Abbad Andaloussi, T. Slaats, A. Burattin, T. T. Hildebrandt, and B. Weber. Evaluating the understandability of hybrid process model representations using eye tracking: First insights. In BPM Workshops, pages 475-481. Springer, 2019.

3. A. Burattin, P. Soffer, D. Fahland, J. Mendling, H. A. Reijers, I. Vanderfeesten, M. Weidlich, and B. Weber. Who is behind the model? classifying modelers based on pragmatic model features. In BPM, pages 322-338, 2018.

4. V. M. Cashen and K. L. Leicht. Role of the isolation effect in a formal educational setting. Journal of Educational Psychology, 61(6p1):484, 1970.

5. F. Chen, J. Zhou, Y. Wang, K. Yu, S. Z. Arshad, A. Khawaji, and D. Conway. Robust Multimodal Cognitive Load Measurement, pages 13-32. Springer, 2016.

6. J. Corbin and A. Strauss. Basics of Qualitative Research: Techniques and Procedures for Developing Grounded Theory. SAGE Publications, 2014.

7. F. Dengler and D. Vrandecic. Wiki-based maturing of process descriptions. In $B P M$, volume 6896 of $L N C S$, pages 313-328. Springer, 2011.

8. D. Fahland, D. Lübke, J. Mendling, H. Reijers, B. Weber, M. Weidlich, and S. Zugal. Declarative versus imperative process modeling languages: The issue of understandability. In BPMDS/EMMSAD, LNBIP, pages 353-366. Springer, 2009.

9. J. Gulden. Visually comparing process dynamics with rhythm-eye views. In $B P M$ Workshops, LNBIP, pages 474-485. Springer, 2016.

10. T. T. Hildebrandt and R. R. Mukkamala. Declarative Event-Based Workflow as Distributed Dynamic Condition Response Graphs. EPTCS, 69:59-73, 2011.

11. H. A. López, S. Debois, T. T. Hildebrandt, and M. Marquard. The process highlighter: From texts to declarative processes and back. In BPM (Dissertation/Demos/Industry), volume 2196 of CEUR, pages 66-70. CEUR-WS.org, 2018.

12. J. Pinggera, T. Porcham, S. Zugal, and B. Weber. LiProMo-Literate process modeling. In CAiSE Forum, volume 855 of $C E U R$, pages 163-170. CEUR-WS.org, 2012 .

13. M. Reichert and B. Weber. Enabling Flexibility in Process-Aware Information Systems. Springer Berlin Heidelberg, Berlin, Heidelberg, 2012.

14. T. Slaats, D. M. M. Schunselaar, F. M. Maggi, and H. A. Reijers. The semantics of hybrid process models. In OTM 2016 Conferences, LNCS. Springer, 2016.

15. H. von Restorff. Über die wirkung von bereichsbildungen im spurenfeld. Psychologische Forschung, pages 299-342, 1933.

16. S. Zugal, J. Pinggera, and B. Weber. The impact of testcases on the maintainability of declarative process models. In BPMDS/EMMSAD, LNBIP, pages 163-177. Springer, 2011. 